\title{
THE FUTURE OF THE JOURNAL OF PRIVACY AND CONFIDENTIALITY
}

\author{
CYNTHIA DWORK
}

Editor-in-Chief, Journal of Privacy and Confidentiality

Harvard University

e-mail address: dwork@seas.harvard.edu

The Journal of Privacy and Confidentiality (JPC) is the only journal to actively solicit contributions from the multi-faceted community of researchers and practitioners for whom privacy is a primary intellectual or operational concern, for dissemination across this broad community. This includes computer scientists, statisticians, lawyers, social scientists, policymakers, health researchers, survey designers, and data-rich corporate players. While not every publication is aimed so broadly, the Journal aims to provide a common forum for all these constituent populations.

The Journal has benefited greatly from Steve Fienberg's generous management style and catholic taste, from the breadth of his interests, and the range of goals to which he applied his prodigious talent and formidable energy. Under his leadership, the Journal operated in an informal style appropriate for launching a fledgling publication. We were not prepared for Steve's loss in 2016 - everything that had facilitated the rapid launching of the Journal and its easy operation was up-ended. We faced problems of staffing, from managing submissions to copy- editing, and of ensuring visibility for past and future published articles. In response, we expanded the responsibilities of the managing editor and were very lucky to have Lars Vilhuber assume this role. Under his superb management we have moved to a platform allowing us to streamline the submission and editorial workflow, and we have arranged for easily visible archival treatment of publications [see Vilhuber, 2018]. We are working to expand our board of editors to handle more, and more broadly drawn, submissions. We are also working to ensure the long- term financial health of the journal, and to create a plan for smooth future transitions.

With the publication of the current issue we re-launch the Journal of Privacy and Confidentiality. We reaffirm our dedication to drawing from multiple disciplines in which privacy and confidentiality are of primary intellectual and operational concern, and to maintaining our status as an open access journal providing a forum for communication across and between these disciplines.

The need for rigorous treatment of privacy and confidentiality in computer science, statistics, ethics, policy, and law has only increased since the founding of the Journal. The past decade has witnessed the large-scale adoption of formal privacy guarantees in industrial

Key words and phrases: editorial, relaunch, Journal of Privacy and Confidentiality, JPC.

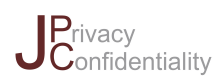

www.journalprivacyconfidentiality.org DOI:10.29012/jpc.708
(C) Cynthia Dwork

(oc) EY-NG-ND Creative Commons (CC BY-NC-ND 4.0) 
settings and by the US Census Bureau. US National Academy of Sciences studies [Groves and Harris-Kojetin, 2017] and federal commissions [CEP, 2018] have noted the application of formal techniques to reducing the response burden in the collection of statistics by the federal statistical agencies and in developing evidence-based policy. The leveraging of personal data in artificial intelligence provides significant avenues for privacy loss, further driving new directions of research in privacy-preserving analytical techniques. We have also seen the exciting application of differential privacy and other information-constraining techniques to preserving validity in exploratory data analysis, when the questions posed are driven by the data themselves and when later studies of a given dataset depend on the results of previous studies on these same data. This scenario will be of increasing relevance in the development of data-driven social science based on the study not only of government administrative data but also of privately held social networking and other data, and we welcome further development of this area. We will continue to solicit high quality work from the constituent communities advancing the state of the art in and across these fields. To this end, we will have a special issue of JPC each year dedicated to the best papers from the annual workshop on Theory and Practice of Differential Privacy (TPDP). Two other planned special issues will address privacy in medical data and privacy in recent law and policy.

Privacy and confidentiality represent just one axis of societal concerns in algorithms and data analysis; others include ensuring representation via improved apportionment, redistricting, and voting schemes; blunting the bias of machine learning algorithms trained on data codifying historical societal biases; mitigating the disparate impact in traditional notions such as signaling mechanisms and the exploration vs. exploitation paradigm; and remediating the fragility of modern machine-learned algorithms in the face of adversarial inputs. Like privacy and confidentiality, these are wide-ranging concerns requiring contributions from, and dissemination of ideas across, multiple disciplines, and from time to time JPC will have special issues dedicated to these and other themes of a similar nature.

Steve Fienberg, Alan Karr, and I founded this Journal to bring together researchers with very different intellectual traditions yet sharing a common goal. The next steps are to grow and broaden the community, and by providing a high quality venue for mathematically rigorous work on privacy and other areas of societal concern, JPC will support the careers of junior researchers entering this emerging discipline.

Cynthia Dwork, Editor-in-Chief, JPC

\section{REFERENCES}

CEP. CEP Final Report: The Promise of Evidence-Based Policymaking, 2018. URL https://www.cep.gov/cep-final-report.html.

R. M. Groves and B. A. Harris-Kojetin, editors. Innovations in Federal Statistics: Combining Data Sources While Protecting Privacy. The National Academies Press, Washington DC, 2017. doi: 10.17226/24652. URL https://www .nap.edu/catalog/24652/.

L. Vilhuber. Relaunching the Journal of Privacy and Confidentiality. Journal of Privacy and Confidentiality, 8(1), dec 2018. doi: 10.29012/jpc.706. URL https://doi.org/10 . 29012/jpc.706. 